"Buscar la verdad y afianzar los valores transcendentales", misión de las universidades en su artículo primero, inspirado en los principios humanísticos. Ley de Universidades 8 de septiembre de 1970.
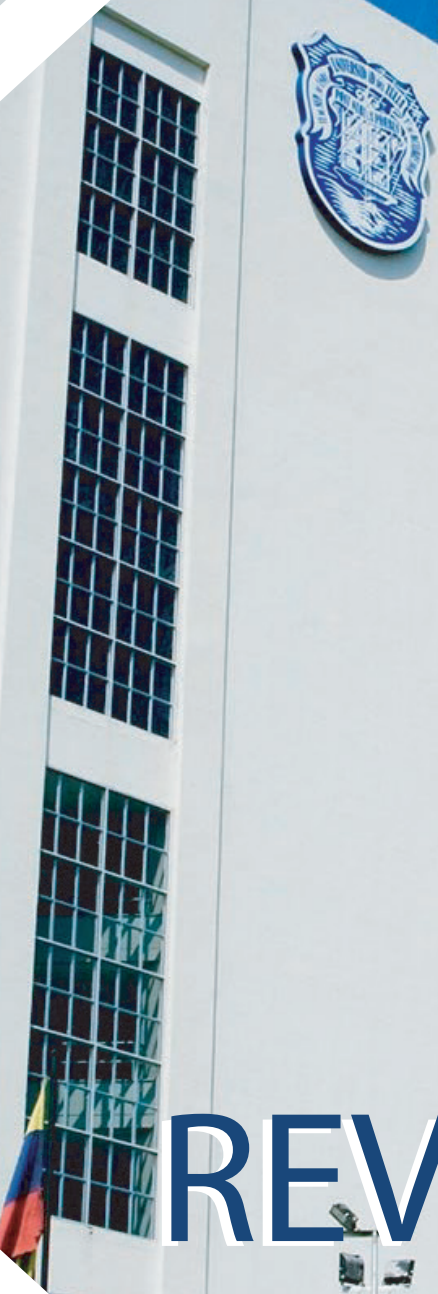

R
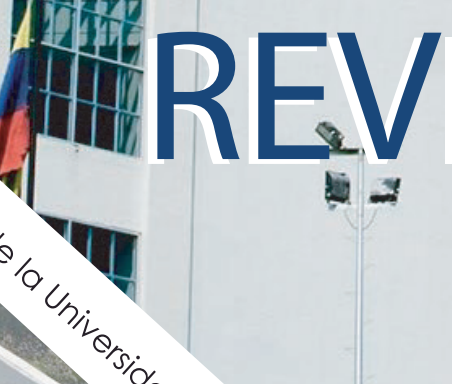

12

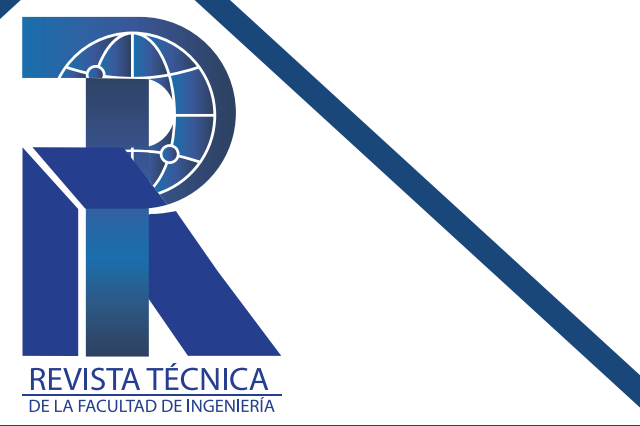

Una Revista Internacional Arbitrada que está indizada en las publicaciones de referencia y comentarios:

- SCOPUS

- Compendex

- Chemical Abstracts

- Metal Abstracts

- World Aluminium Abstracts

- Mathematical Reviews

- Petroleum Abstracts

- Current Mathematical Publications

- MathSci

- Revencyt

- Materials Information

- Periódica

- Actualidad Iberoamericana 
Rev. Téc. Ing. Univ. Zulia. Volumen Especial, 2020, No. 2, 19-25

\title{
Influence of on-site curing on the compressive strength of concrete
}

\author{
W. Stalin Alcívar ${ }^{*} \mathbb{D}^{\mathbb{D}}$, Yordy Mieles Bravo1${ }^{\mathbb{D}}$, Christian Pavón ${ }^{2} \mathbb{D}$, Edwin Solórzano $^{1} \mathbb{D}$, \\ Leidy Palacios ${ }^{1} \mathbb{D}$
}

${ }^{1}$ Departamento de Construcciones Civiles, Facultad de Ciencias Matemáticas Físicas y Químicas, Universidad Técnica de Manabí, Portoviejo, Ecuador.

${ }^{2}$ Licenciatura en Físico-Matemáticas, Universidad de Guayaquil, Ecuador.

*Autor de correspondencia: wsalcivar@hotmail.com

https://doi.org/10.22209/rt.ve2020n2a03

Recepción: 21/02/2020 | Aceptación: 22/05/2020 | Publicación: 31/07/2020

\begin{abstract}
The compressive strength of concrete is of vital importance in the behavior of the structuresand is dependent on mixing and curing on site. Different curing conditions that are far from the ideal and proven laboratory conditions can be observed in the constructions.In the present investigation, the compressive strength of concrete is determined through tests of cylindrical specimens subjected to different curing methods: immersion, use of a membrane-forming compound, use of plastic cellophane, spray hydration (three times a day)b and outdoors (without curing), determining the influence of the curing method on compressive strength at different ages. For each curing method, 6 specimens were prepared which were arranged to be tested at ages 7, 14, and 28 days, two specimens by age. Compressive strength at 28 days was obtained with the immersion cure method. Among the on-site curing methods, the periodic hydration curing method delivered greater compressive strength versus cure with the use of membrane-forming curing compounds or the use of the plastic coating.
\end{abstract}

Keywords: Concrete curing; Concrete; Compressive strength.

\section{Influencia del curado en obra sobre la resistencia a la compresión del concreto}

\begin{abstract}
Resumen
La resistencia a la compresión del concreto resulta de vital importancia en el comportamiento de las estructuras, y es dependiente de la mezcla y el curado en obra. En las construcciones se pueden observar diferentes condiciones de curado que distan de las condiciones ideales y comprobadas de laboratorio. En la presente investigación se determina la resistencia a la compresión del concreto mediante ensayos de probetas cilíndricas sometidas a diferentes métodos de curado: inmersión, uso de un compuesto formador de membrana, uso de celofán plástico, hidratación mediante aspersión (tres veces al día) y a la intemperie (sin curado), determinándose la influencia del método de curado en la resistencia a la compresión a diferentes edades. Para cada método de curado se elaboraron 6 probetas las cuales fueron dispuestas a ser ensayadas en edades de 7, 14 y 28 días, dos probetas por edad. La resistencia a la compresión a los 28 días se obtuvo con el método de curado mediante inmersión. Entre los métodos de curado que se realizan en obra, el método de curado por hidratación periódica entregó mayor resistencia a la compresión frente al curado con el uso de curadores formadores de membranas o el uso de revestimiento plástico.
\end{abstract}

Palabras clave: Curado; concreto; resistencia a la compresión. 


\section{Introducción}

La calidad del concreto usualmente se evalúa en función de su resistencia característica a la compresión (f'c), la cual debe estar acorde con la especificada por el diseñador estructural de la obra. Varias investigaciones [1-3] han documentado que tanto la resistencia a la compresión como la durabilidad del concreto se desarrollan sólo si este se somete a un adecuado proceso de curado. El curado del concreto es definido por el Comité 308 del American Concrete Institute [4] como el proceso que consiste en mantener un contenido satisfactorio de humedad y temperatura en el concreto recién colocado para que se puedan desarrollar las propiedades deseadas, y respecto a su importancia, la Portland Cement Association [5] enfatiza que este tiene una fuerte influencia sobre las propiedades del concreto endurecido tales como la durabilidad, resistencia, permeabilidad y la resistencia a la abrasión. En términos prácticos se debe controlar la temperatura y evitar o reducir las pérdidas de agua del concreto en el periodo comprendido entre su colocación y los siguientes 14 días [6].

Las condiciones en que se realiza el curado en obra de elementos estructurales, tales como losas, vigas y columnas, que forman parte de una edificación, difieren de las condiciones ideales de laboratorio [7]. Sin embargo, estos elementos deben desarrollar resistencias de diseño, ante esfuerzos axiales, de flexión, de corte y torsión, y estás resistencias son calculadas usando propiedades mecánicas características tanto para el concreto como para el acero de refuerzo [8].

En el Ecuador, el curado del concreto de las columnas, usualmente se realiza mediante el rocío de agua de tres o cuatro veces al día, especialmente los primeros días después del desencofrado, y en otros casos se realiza mediante el empleo de algún compuesto de curado formador de membrana. El revestimiento de los elementos de concreto armado en estado fresco con celofán plástico para evitar la evaporación del agua del curado es de reciente uso en el medio ecuatoriano, figura 1(a). Este método de curado genera dudas en cuanto a su efectividad sobre la evaporación del agua a través de los traslapes del celofán, aunado a las roturas ocasionales del plástico debido a accidentes por procesos constructivos, como se muestra en la figura 1(b).

Diferentes estudios [9-12] se han orientado a estimar la resistencia a la compresión que se obtiene al emplear diferentes métodos o condiciones de curado en cuanto a temperatura y humedad se refiere. Los datos experimentales del concreto curado con el uso de plástico son prácticamente nulos en el Ecuador, y en general, en la literatura técnica se encuentra poca información que respalde su uso. Esto motivó a realizar una serie de ensayos experimentales para estimar la influencia del método de curado en la resistencia a la compresión a diferentes edades del concreto, usando los métodos actualmente usados en el curado de columnas en el Ecuador; finalmente se contrastan los resultados con los ya documentados en diversos trabajos anteriores [13-15].

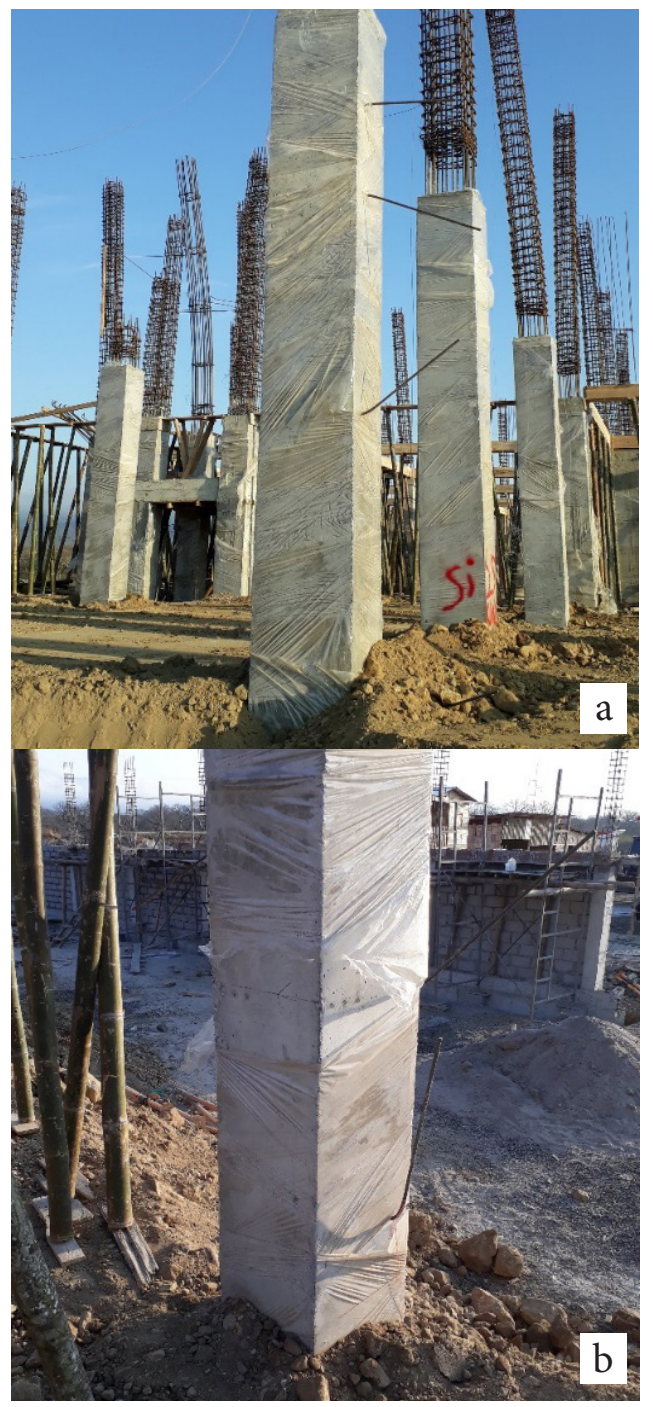

Figura 1. (a) Curado con celofán plástico en columnas. (b) Rotura accidental del plástico.

\section{Metodología}

Se determina la resistencia a la compresión del concreto mediante ensayos de probetas cilíndricas (de $10 \mathrm{~cm}$ de diámetro por $20 \mathrm{~cm}$ de altura) sometidas a cuatro (04) diferentes métodos o condiciones de curado: inmersión, adición de compuesto de curado formador de membrana, uso de celofán de plástico, hidratación periódica (rociado tres veces al día) y a la intemperie (sin 
curado), y se determina la influencia del método de curado en la resistencia a la compresión a diferentes edades, 7 , 14 y 28 días, figura 2(a). Se elaboraron 30 probetas en total, 6 probetas para cada método de curado, dos de ellas ensayadas en cada fecha. Se realizó el diseño de la mezcla, de acuerdo con el método del ACI 211 [16], para lograr una resistencia a la compresión, $f^{\prime} c$, de $24 \mathrm{MPa}$, y la dosificación usada se muestra en la Tabla 1. En la elaboración de las probetas se cumplen las especificaciones dadas por la ASTM C31 [7] y los ensayos de resistencia a la compresión se basan en la norma ASTM C873 [17]. cual resultó igual a $160 \mathrm{~mm}$, figura 2(c), valor inferior al asentamiento estimado en el diseño de la mezcla el cual era de $200 \mathrm{~mm} \pm 40 \mathrm{~mm}$.

\section{Inmersión}

Es ampliamente conocido que este método de curado produce los mejores resultados en la resistencia a la compresión del concreto, sin embargo, presenta inconvenientes de tipo práctico ya que este método requiere sumergir completamente el elemento de

Tabla 1 Dosificación de la mezcla de concreto utilizada.

\begin{tabular}{|c|c|c|c|c|}
\hline \multicolumn{5}{|c|}{ Diseño $24 \mathrm{MPa}$ a los 28 días } \\
\hline \multirow{2}{*}{ Descripción } & \multicolumn{4}{|c|}{ Dosificación 1 m $^{3}$} \\
\hline & Pesos (kg) & $\%$ & Densidades $\left(\mathrm{kg} / \mathrm{m}^{3}\right)$ & Volumen $\left(\mathrm{m}^{3}\right)$ \\
\hline Agregado grueso $(1 / 2 \mathrm{plg})$ & 1063 & 56,09 & 2785 & 0,3817 \\
\hline Agregado fino (arena) & 832 & 43,91 & 2634 & 0,3159 \\
\hline Cemento & 360 & & 3000 & 0,1200 \\
\hline Aditivo líquido retardante. & 3,20 & & 1140 & 0,0028 \\
\hline Aditivo líquido plastificante & 2,10 & & 1180 & 0,0018 \\
\hline Agua & 219 & & 1000 & 0,2190 \\
\hline $\begin{array}{c}\text { Peso teórico } \\
\text { Volumen }\left(\mathrm{m}^{3}\right)\end{array}$ & 2479 & $\mathbf{a} / \mathbf{c}$ & 0,61 & 1,0410 \\
\hline
\end{tabular}

Se utilizó cemento portland puzolánico tipo IP como aglomerante, los agregados finos y gruesos son provenientes de Colimes, provincia del Guayas, y de Montecristi, provincia de Manabí, respectivamente. Tanto el agregado grueso como el agregado fino usado en la mezcla cumple con la normativa ASTM C33 [18]. El diseño de la mezcla utilizada en la investigación contempla el uso de un aditivo líquido retardante y de un aditivo líquido plastificante que cumplen con las especificaciones de la ASTM C494 [19], para los tipos B y F, respectivamente. La mezcla corresponde a un diseño típico de los concretos comerciales en la localidad.

Previo a la confección de las probetas para el ensayo de resistencia a la compresión, se comprobó la temperatura de la mezcla y la trabajabilidad. Se midió la temperatura con un termómetro colocado en la muestra durante un tiempo aproximado de dos minutos, hasta que el registro de la temperatura fue constante, como lo indica la norma ASTM C1064 [20]. La figura 2(b) muestra la temperatura registrada en la mezcla, la cual fue de $29^{\circ} \mathrm{C}$, aceptable de acuerdo con la especificación del ACI 301 [21], la cual limita la temperatura máxima del concreto a $35^{\circ} \mathrm{C}$ en el momento de la colocación. La trabajabilidad se la midió mediante el asentamiento de cono de Abrams, basado en la norma ASTM C143 [22]. La distancia comprendida entre la posición inicial de la mezcla y la desplazada determina el valor del asentamiento, el concreto, lo cual resulta complejo realizar en obra. Seis de las probetas confeccionadas fueron curadas a nivel de laboratorio por inmersión en piscina de agua saturada con hidróxido de calcio, ver figura 3(a), a una temperatura promedio de $20^{\circ} \mathrm{C}$ [7]. Las probetas fueron retiradas de la piscina 30 minutos antes de ser ensayadas.

\section{Uso de compuesto de curado formador de membrana}

Seis de las probetas confeccionadas se curaron mediante la aplicación de un compuesto de curado formador de membrana, el cual forma sobre el concreto una película delgada, elástica e impermeable al agua y al aire, ver figura 3(b). Se aplicó una emulsión de parafina diluida en agua, en proporciones 1:3, sobre el concreto fresco en la cara superior de las probetas e inmediatamente después de desmoldar las probetas en el perímetro y cara inferior.

\section{Revestimiento con celofán de plástico}

El uso de plástico como recubrimientos en métodos de curado se presenta en mayor porcentaje en columnas, existiendo preferencia por el uso de plástico en zonas donde predominan las altas temperaturas [23]. Seis probetas fueron cubiertas, después de ser desmoldadas, con una lámina plástica transparente, tal como se muestra en la figura 4(a), la misma que es recomendada para climas cálidos. Se recubrió la parte inferior y lateral de las probetas, con la excepción de la parte superior, simulando la forma de aplicación en una columna. 


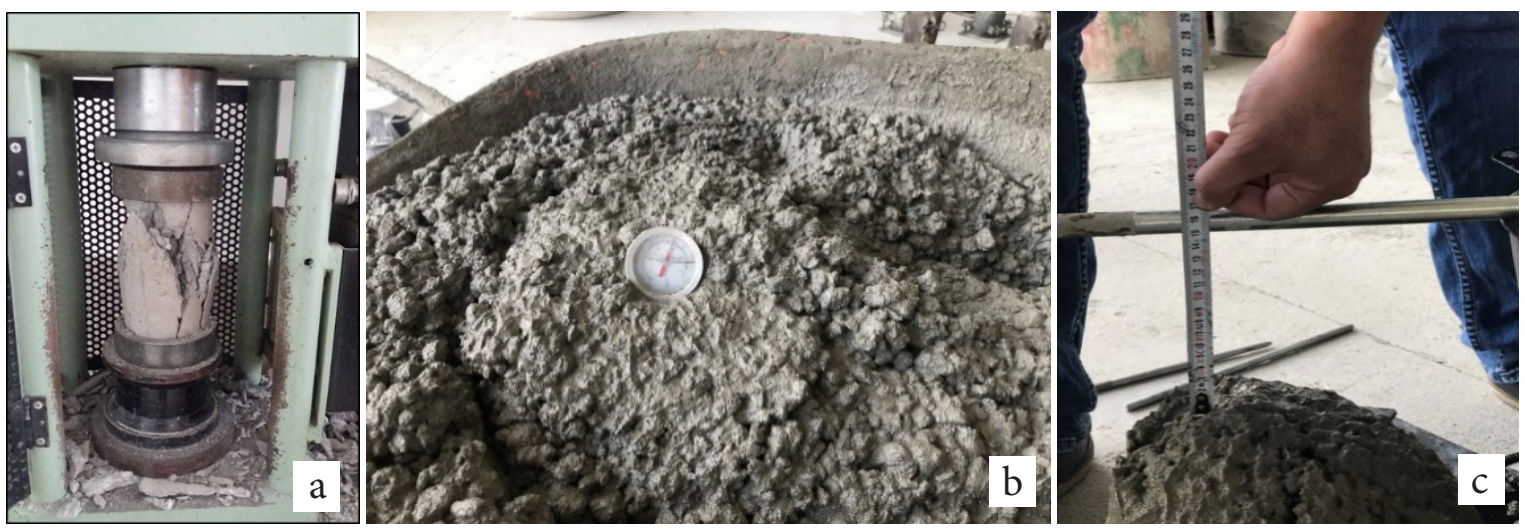

Figura 2. (a) Rotura de probeta a la compresión; (b) Medición de la temperatura de la mezcla; (c) Medición del asentamiento de cono de Abrams.
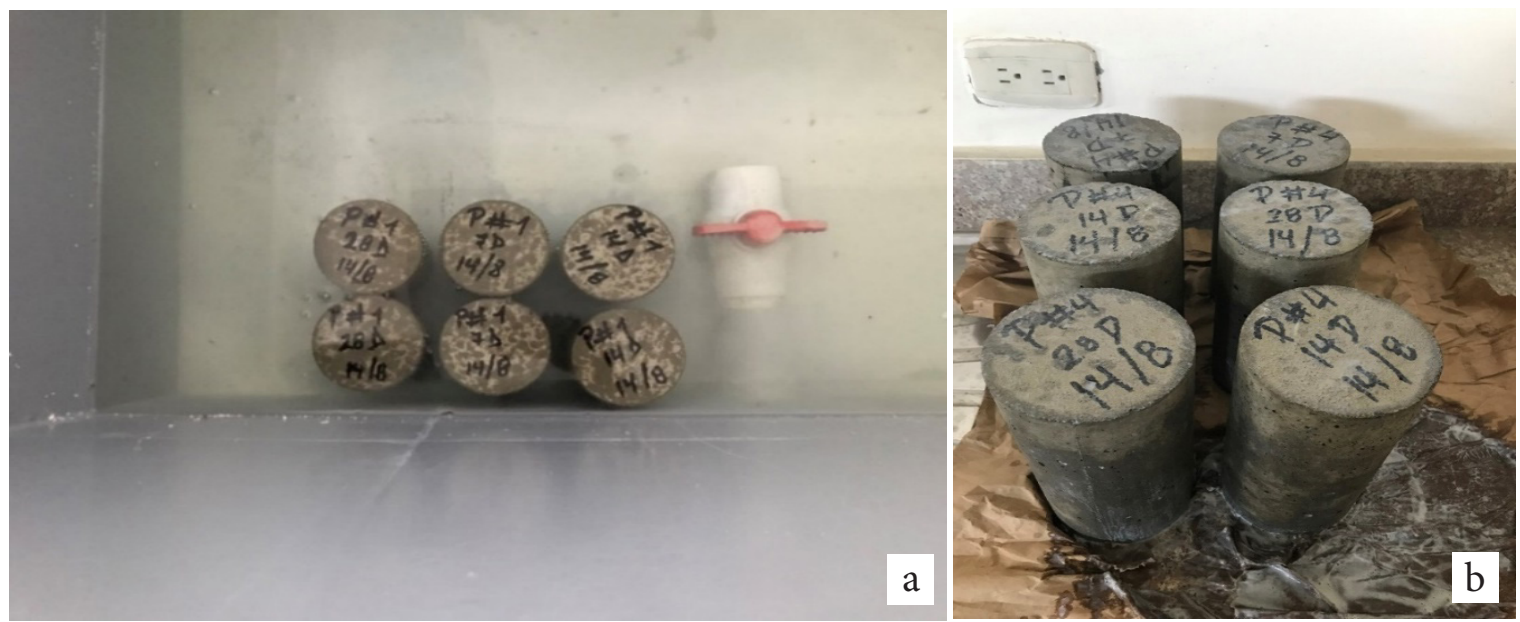

Figura 3. (a) Probetas sumergidas en la piscina. (b) Probetas curadas con aplicación de compuesto curador.
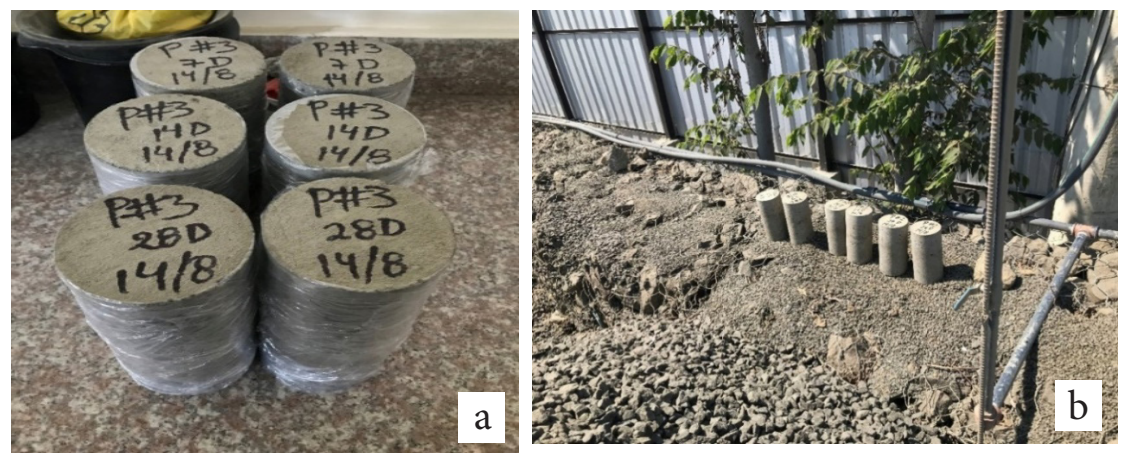

Figura 4. (a) Probetas revestidas con plástico. (b) Probetas curadas con hidratación periódica. (c) Muestras a la intemperie, sin curado. 


\section{Hidratación periódica}

Seis de las probetas fueron curadas mediante hidratación por aspersión con manguera 3 veces al día, imitando lo que sucede en obra, como ilustra la figura 4(b). Para la hidratación diaria con agua potable se consideraron los siguientes horarios: primera hidratación por la mañana, 8:00am, segunda hidratación una vez al medio día, 12:30pm, y la tercera hidratación al término de la jornada de trabajo 17:00pm.

\section{Intemperie}

Seis de las probetas confeccionadas se dejaron al aire libre sin aplicar ningún método de curado como se muestra en la figura 4(c). El lugar donde se ubicaron las muestras se encontraba expuesto al sol y aislado de tal manera que no se vea afectada la integridad de las muestras.

\section{Resultados y Discusión}

La mezcla del concreto fue diseñada para alcanzar una resistencia a la compresión superior a los $24 \mathrm{MPa}$ a los 28 días en condiciones de laboratorio. Por las características propias del diseño del concreto, la resistencia a la compresión a los 28 días resultó superior a los $24 \mathrm{MPa}$ en las probetas curadas bajo los diferentes métodos de curado. Las probetas fueron ensayadas a edades de 7, 14 y 28 días. En el caso de las probetas a la intemperie, sin ningún método de curado, la resistencia a la compresión resultó ligeramente inferior a los $24 \mathrm{MPa}$, (ver la figura 5). La resistencia máxima a la compresión a los 28 días fue el método de curado por inmersión bajo condiciones ideales de laboratorio e igual a $32,16 \mathrm{MPa}$, mientras que la mínima resistencia fue de 23,29 MPa obtenida para las probetas sin curado, indicados en la tabla 2.

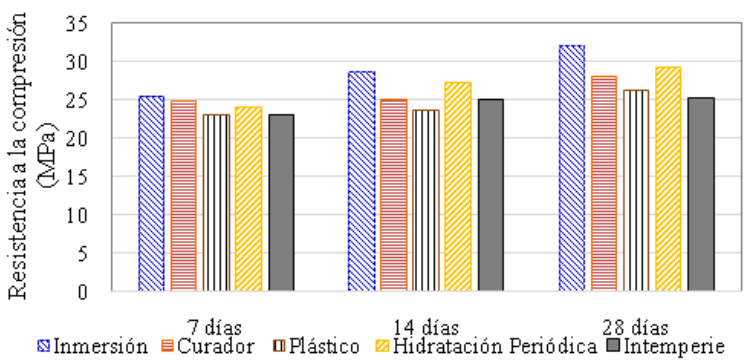

Figura 5. Resistencia a la compresión a diferentes edades en días

La diferencia de resistencia a la compresión obtenida entre las probetas curadas por inmersión y las probetas sin ningún tratamiento de curado resultó ser relativamente baja a los 7 días, 2,52 $\mathrm{MPa}$; pero se hizo más evidente a los 28 días, en que se obtuvo una diferencia de 6,87 $\mathrm{MPa}$ entre la resistencia a compresión de las probe- tas curadas por inmersión y las probetas sin ningún tratamiento de curado.

Para poder verificar que existen diferencias estadísticamente significativas entre los distintos métodos de curado, se realizó una prueba chi-cuadrada para asociación para las ganancias porcentuales de 7 a 14 y de 14 a 28 días, con un nivel de significancia de 0,01; figura 6 .

a

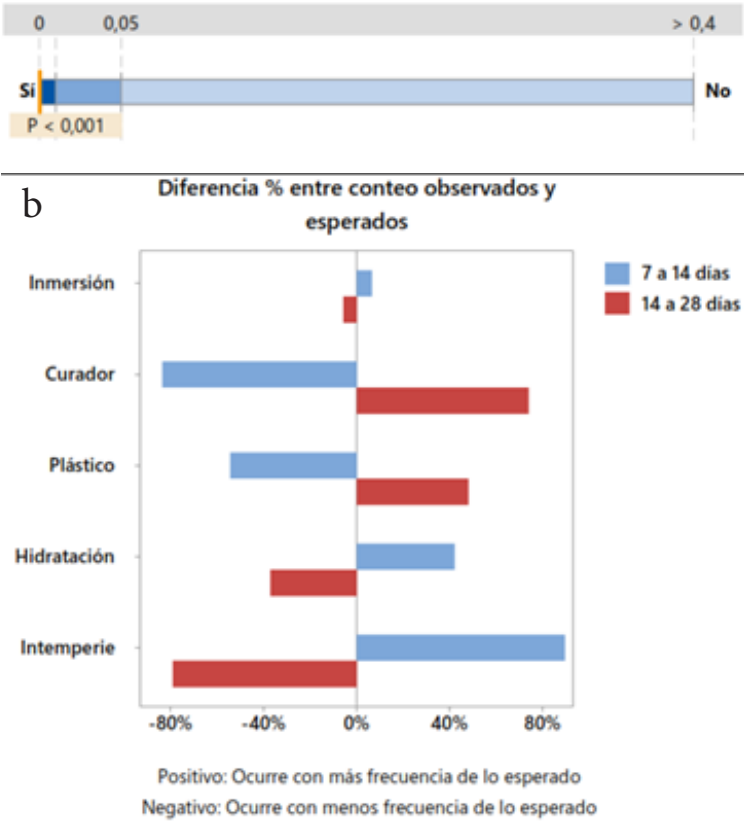

Figura 6. Resultados dela prueba chi-cuadrada para asociación.(a) Valor del estadístico P.(b) Diferencia porcentual entre conteo observado y esperado.

Basados en el valor $p<0,001$ observado en la figura 6(a), se puede concluir que existen diferencias entre los perfiles porcentuales de resultados con un nivel de significancia de 0,01; es decir, la resistencia a la compresión obtenida difiere significativamente dependiendo del tratamiento aplicado. Adicionalmente, en la figura 6(b), se observa que, en los métodos de inmersión, hidratación e intemperie, la resistencia a la compresión se incrementa más de lo esperado en los primeros 14 días. Mientras que en el método de uso de curador y en el uso de plástico la resistencia a la compresión se incrementa más de lo esperado en un intervalo de 28 días. En todos los métodos se observó un aumento de resistencia con las diferentes edades analizadas, como se aprecia en la figura 7.

Se obtuvo las mayores resistencias a compresión en las probetas curadas en condiciones de laboratorio. El curado por hidratación periódica en los 28 días permitió alcanzar una resistencia de compresión, 29,30 MPa, supe- 
Tabla 2. Resistencia a la compresión de los distintos métodos de curado, en MPa.

\begin{tabular}{cccccc}
\hline Tipo de curado & 7 días & 14 días & $\begin{array}{c}\text { Diferencia \% } \\
\text { de 7 a 14 días }\end{array}$ & 28 días & $\begin{array}{c}\text { Diferencia \% de } \\
14 \text { a 28 días }\end{array}$ \\
\hline Inmersión & 25,57 & 28,71 & 12 & 32,16 & 12 \\
Curador & 24,81 & 25,16 & 1 & 28,08 & 7 \\
$\begin{array}{c}\text { Plástico } \\
\text { Hidratación perió- } \\
\text { dica }\end{array}$ & 23,05 & 23,65 & 3 & 26,37 & -7 \\
Intemperie & 23,05 & 24,32 & 14 & 29,30 & 11 \\
\hline
\end{tabular}

rior a la obtenida con el uso de curadores formadores de membrana y el uso de revestimientos de plástico. En las probetas curadas por medio de la aplicación de curador formador de membrana, el cual no es de uso frecuente en la construcción local, alcanzó una resistencia a la compresión de 28,08 MPa a los 28 días, inferior a la obtenida mediante la hidratación periódica y superior a la obtenida mediante el uso de revestimiento de plástico. En las probetas curadas con el uso de revestimiento plástico se obtuvo una resistencia a la compresión, a los 28 días de 26,37 MPa. Para el caso de las probetas que no fueron curadas, a la intemperie, se obtuvo una resistencia a la compresión a los 28 días de 25,29 $\mathrm{MPa}$, inferior a lo obtenido al aplicar alguno de los métodos de curado, aunque superior a los 24 MPa definidos en el diseño de la mezcla.

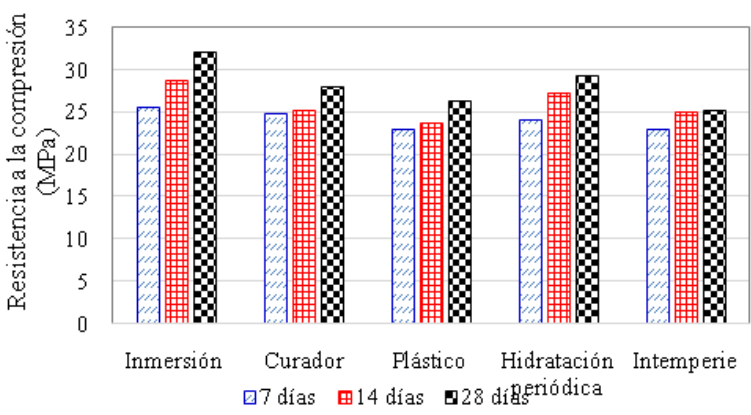

Figura 7. Resistencia a la compresión a diferentes edades en días por método de curado

Los resultados comprueban que el método de curado influye directa y significativamente en la resistencia a la compresión final del concreto. En cuanto a la resistencia a la compresión en el concreto, se pudo determinar que los métodos de curado que se realizan incorporando directamente agua al concreto, como la inmersión o la hidratación periódica, resultaron más efectivos ante los métodos de curado con curadores formadores de membrana y uso de revestimiento plástico. La práctica actual de la construcción en el Ecuador ha incorporado el método de curado de columnas mediante el revestimiento plástico, sin embargo, con este método no se obtuvieron resisten- cias superiores, en ninguna de las edades, a las alcanzadas por hidratación periódica de las probetas. Los resultados mostrados concuerdan con los presentados por Jackson et. al. [14] en cuanto a la poca eficiencia del método de curado con el uso del revestimiento plástico frente al método de curado por hidratación; en un estudio realizado por Bushlaibi [15]se destaca el uso del método de curado con revestimiento plástico frente al curado por hidratación periódica, en dicho estudio las probetas fueron hidratadas mediante el rocío de agua dos veces al día, lo cual infiere que en la comparación de ambos métodos de curado se debe considerar las veces en que se hidrata la probeta, y sería conveniente realizarlo un estudio comparativo posterior en que se introduzca esto como variable.

\section{Conclusiones}

El método de curado por hidratación periódica resultó más eficiente, en cuanto a resistencia a la compresión se refiere, frente al método de curado con el uso de curadores formadores de membrana o el uso de revestimiento con plástico.

El método de curado mediante el revestimiento con plástico alcanzó resistencias a la compresión del concreto inferiores a las obtenidas con el uso de curadores formadores de membranas y con el uso de la hidratación periódica, en todas las edades. No se recomienda por lo tanto en obra el curado con revestimiento plástico, siendo el método de hidratación periódica la mejor opción en obra.

\section{Agradecimientos}

A la empresa Hormigonera Portoviejo por las facilidades brindadas para llevar adelante esta investigación.

\section{Referencias Bibliográficas}

[1] Gonnerman, H. and ShumanE.: Flexure and tension tests of plain concrete. Report of the Director of Research, Port. Cem. Assoc. (1928) 149-163. 
[2] Hernández, N.: Efecto del curado sobre un concreto de resistencia de diseño de $210 \mathrm{~kg} / \mathrm{cm} 2$. Revista Ingenieria UC, Vol. 17, No. 3 (2010) 92-96.

[3] Rojas, E.R., L.F.H. Mora, and F.d.M.M. Umaña: Resistencia a compresión versus tiempo de curado en concreto hidráulico a partir de cementos modificados. Métodos y Materiales,Vol. 9(2019)110.

[4] Committee 308, ACI.: ACI 308R-16; Guide to External Curing of Concrete. American Concrete Institute, Farmington Hills, Mich, 2016.

[5] Portland Cement Association: Diseño y control de mezclas de concreto. Portland Cement Association, 1a edición, Capítulo, 2004. 3, 77-91.

[6] Fernández Luco, L.: Propuesta de indicadores de la eficacia del curado en obra. Concreto y cemento. Investigación y desarrollo, Vol. 1, No. 2(2010) 1729.

[7] ASTM C31/ C31M-19a: Standard Practice for Making and Curing Concrete Test Specimens in the Field, ASTM International, Wet Conshohocken, PA, 2019.

[8] Committee 318, ACI: Building code requirements for structural concrete (ACI 318-19) and commentary, American Concrete Institute, 2019.

[9] Zeyad, A.M.: Effect of curing methods in hot weather on the properties of high-strength concretes. Journal of King Saud University-Engineering Sciences, Vol. 31, No. 3 (2019) 218-223.

[10] Raheem, A.A., A.A. Soyingbe, and A.J. Emenike: Effect of curing methods on density and compressive strength of concrete. International Journal of Applied Science and Technology, Vol. 3, No. 4 (2013)

[11] Ibrahim, M., et al.: Effect of curing methods on strength and durability of concrete under hot weather conditions. Cement and Concrete Composites, Vol. 41 (2013) 60-69.

[12] Nematollahzade, M., et al.: Influence of different curing conditions and water to cement ratio on properties of self-compacting concretes. Construction and Building Materials, Vol.237
(2020) 117570.

[13] Princy, K. and D.E. John: Study on the Effectiveness of Various Curing Methods on the Properties of Concrete. International Journal of Engineering Research And, Vol. 4 (2015).

[14] Jackson, E.N. and B.B. Akomah: Comparative Analysis of The Strength of Concrete With Different Curing Methods In Ghana. The International Journal of Engineering and Science (IJES), Vol. 7, No. 9 (2018) 39-44.

[15] Bushlaibi, A.: Effects of environment and curing methods on the compressive strength of silica fume high-strength concrete. Advances in cement research, Vol. 16, No. 1 (2004) 17-22.

[16] Committee 211, ACI.: ACI 211.1-91; Standard practice for selecting proportion for normal, heavy weight and mass concrete. ACI Manual of Concrete Practice. Part 1(1996).

[17] ASTM C873 / C873M-10: Standard test method for compressive strength of concrete cylinders cast in place in cylindrical molds. C873, West Conshohocken, PA, 2010.

[18] ASTM C33 / C33M-18: Standard Specification for Concrete Aggregates, ASTM International, West Conshohocken, PA, 2008.

[19] ASTM C494: Standard specification for chemical admixtures for concrete, ASTM International, West Conshohocken, PA, 2004.

[20] ASTM C1064 / C1064M-17: Standard Test Method for Temperature of Freshly Mixed Hydraulic Cement Concrete, ASTM International, West Conshohocken, PA, 2017.

[21] Committee 301, ACI: ACI 301-16; Specifications for Structural Concrete,American Concrete Institute, 2008.

[22] ASTM C143 / C143M-15a: Standard Test Method for Slump of Hydraulic-Cement Concrete, ASTM International, West Conshohocken, PA, 2015.

[23] Medina-Sierra, W.A.: Prácticas en el Curado del Concreto, Caso de Estudio Tunja. Ingenio Magno, Vol. 5, No. 1 (2014) 61-71. 


\section{REVISTA TECNICA}

DE LA FACULTAD DE INGENIERIA

UNIVERSIDAD DEL ZULIA

Volumen Especial, 2020, No. 2, pp. 04 - 110

Esta revista fue editada en formato digital y publicada en Julio de 2020, por el Fondo Editorial Serbiluz, Universidad del Zulia. Maracaibo-Venezuela

www.luz.edu.ve

www.serbi.luz.edu.ve

www.produccioncientifica.org 